\title{
Fonctions de Morse sur les espaces exotiques.
}

\author{
G. VRayceanu (Bucarest)
}

Summary. - It is shown that on an exotique space $\nabla_{2 n-1}$ of Kervaire type, we can construct a Morse function having only four critical points, the maximum, the minimum and two points of index $n-1$ and $n$.

Etant donnée la sphère $S_{2 n+1}$ dans l'espace euclidien $E_{2 n+2}\left[x_{0}, \ldots, x_{n}, y_{0}, \ldots, y_{n}\right]$ par l'équation

$$
z_{0} \bar{z}_{0}+\ldots+z_{n} \bar{z}_{n}=2 \quad\left[z_{h}=x_{h}+i y_{h}, h=0, \ldots, n\right],
$$

on peut considerer les variétés $V_{2 n-1}$ definies sur $S_{2 n+1}$ par l'équation

$$
z_{0}^{k}+z_{1}^{2}+\ldots+z_{n}^{2}=0
$$

où $k$ est un nombre entier plus grand que 1 . Si $k=2$ les variétés $V_{2 n-1}$ sont des variétés quadratiques

$$
\begin{aligned}
& x_{0}^{2}+\ldots+x_{n}^{2}=1 \\
& x_{0} y_{0}+\ldots+x_{n} y_{n}=0 \\
& y_{0}^{2}+\ldots+y_{n}^{2}=1,
\end{aligned}
$$

Si $k$ est un nombre impair, les variétés $V_{2 n-1}$ sont des sphéres standard ou exotiques du type Kervaire [1]. On peut done dire que les espaces (1), (2) pour $k$ un nombre entier plus grand que 1 sont des espaces exotiques, où quasi-quadratiques.

Nous allons montrer ici que l'on peut construire des fonctions de Morse sur les $V_{2 n-1}$ exotiques, qui ont seulement quatres points critiques.

Pour cela observons que nous avons

$$
z_{h}^{2}=x_{h}^{2}-y_{h}^{2}+2 i x_{h} y_{h}, \quad z_{0}^{k}=P+i Q \quad(h=1, \ldots, n)
$$

où $P ; Q$ sont des polynomes homogènes du dégré $k$

$$
\begin{aligned}
& P=x_{0}^{k}-\frac{k(k-1)}{2} x_{0}^{k-2} y_{0}^{2}+\ldots \\
& Q=k x_{0}^{k-1} y_{0}+\ldots
\end{aligned}
$$

(*) Entrata in Redazione il 20 ottobre 1972 .

3 - Annali di Matematica 
et ces polynomes satisfont évidemment aux conditions de Cauchy

$$
P_{x_{0}}=Q_{y_{0}}, \quad P_{y_{0}}=-Q_{x_{0}}
$$

Cela dit, on peut écrire les trois équations réelles de $V_{2 n-1}$ sous la forme

$$
\begin{aligned}
& R_{1}=x_{0}^{2}+y_{0}^{2}+P+2 x_{h}^{2}-2=0 \\
& R_{2}=x_{0}^{2}+y_{0}^{2}-P+2 y_{h}^{2}-2=0 \\
& R_{3}=Q+2 x_{h} y_{h}=0 .
\end{aligned}
$$

Considérons alors sur $V_{2 n-1}$ comme fonction de Morse

$$
F^{\prime}=4 x_{1}+4 m y_{2}+2 a x_{0}
$$

où $m$ est une constante différente de zéro et $a$ une constante quelquonque et cherchons à voir quelles sont les points critiques de cette fonction. Pour cela on peut utiliser la fonction auxiliaire

$$
\varphi=F-\lambda R_{1}-\mu R_{2}-2 \varrho R_{3}
$$

où $\lambda, \varrho, \mu$ sont des parametres. If faut done pour un point critique de $F$ sur $V_{2 n-1}$ que toutes les dérivées de cette fonction par rapport aux coordonnées de $E_{2 n+2}$ soient nulles.

En dérivant par rapport aux coordonnées $x_{0}, y_{0}$, nous obtenons les deux équations

$$
\begin{aligned}
& \lambda\left(2 x_{0}+P_{x_{0}}\right)+\mu\left(2 x_{0}-P_{x_{0}}\right)+2 \varrho Q_{x_{0}}=2 a, \\
& \lambda\left(2 y_{0}+P_{v_{0}}\right)+\mu\left(2 y_{0}-P_{v_{0}}\right)+2 \varrho Q_{y_{0}}=0 .
\end{aligned}
$$

Si nous dérivons par rapport aux $x_{1}, y_{1}$ et $x_{2}, y_{2}$ nous obtenons

$$
\begin{array}{ll}
\lambda x_{1}+\varrho y_{1}=1, & \varrho x_{1}+\mu y_{1}=0, \\
\lambda x_{2}+\varrho y_{2}=0, & \varrho x_{2}+\mu y_{2}=m .
\end{array}
$$

Quant aux dérivées par rapport aux autres variables, elles nous donnent

$$
\lambda x_{j}+\varrho y_{j}=0, \quad \varrho x_{j}+\mu y_{j}=0 \quad(j=3, \ldots, n)
$$

Les équations $\left(5^{\prime}\right)$ nous disent que l'on doit avoir

$$
\lambda \mu-\varrho^{2} \neq 0
$$


En effet les équations en $x_{1}, y_{1}$ de $\left(5^{\prime}\right)$ peuvent n'être pas indépendantes seulement, si $\varrho=\mu=0$, ce qui est en contradiction avec la dernière équation $\left(5^{\prime}\right)$. De même, les équations $\left(5^{\prime}\right)$ nous disent que l'on a

$$
x_{1} y_{2}-x_{2} y_{1} \neq 0
$$

et l'on peut résoudre ces équations par rapport aux variables $x_{2}, \lambda, \varrho, \mu$ par les formules

$$
x_{2}=m y_{1}, \quad \lambda=\frac{y_{2}}{x_{1} y_{2}-m y_{1}^{2}},
$$

$$
\varrho=-\frac{m y_{1}}{x_{1} y_{2}-m y_{1}^{2}}, \quad \mu=\frac{m x_{1}}{x_{1} y_{2}-m y_{1}^{2}} \text {. }
$$

En tenant compte de la $\left(6^{\prime}\right)$, les équations (6) nous donnent

$$
x_{j}=y_{j}=0 \quad(j=3, \ldots, n)
$$

donc les équations (3) s'écrivent en tenant compte de la première (7)

$$
\begin{aligned}
& x_{0}^{2}+y_{0}^{2}+P+2\left[x_{1}^{2}+m^{2} y_{1}^{2}\right]=2 \\
& x_{0}^{2}+y_{0}^{2}-P+2\left[y_{1}^{2}+y_{2}^{2}\right]=2 \\
& Q+2 y_{1}\left[x_{1}+m y_{2}\right]=0
\end{aligned}
$$

De même, les équations (5) s'écrivent en tenant compte des (7)

$$
\begin{aligned}
& y_{2}\left[2 x_{0}+P_{x_{0}}\right]+m x_{1}\left[2 x_{0}-P_{x_{0}}\right]-2 m y_{1} Q_{x_{0}}=2 a\left[x_{1} y_{2}-m y_{1}^{2}\right] \\
& y_{2}\left[2 y_{0}+P_{y_{0}}\right]+m x_{1}\left[2 y_{0}-P_{v_{0}}\right]-2 m y_{1} Q_{v_{0}}=0 \\
& x_{1} y_{2}-m y_{1}^{2} \neq 0 .
\end{aligned}
$$

Il en résulte le théoreme:

Les points critiques de la fonction (4) sont les solutions en $x_{0}, y_{0}, x_{1}, y_{1}, y_{2}$ des équations (9) et (10), les $x_{j}, y_{j}(j>2)$ étant nulles, et $x_{2}$ est donnée par la prémière (7).

Or supposons que l'on a

$$
x_{0}=y_{0}=0,
$$

ce qui peut arriver seulement si la constante $a$ est nulle.

En ce cas les (10) sont vérifiées et les (9) s'écrivent

$$
x_{1}^{2}+m^{2} y_{1}^{2}=1, \quad y_{1}^{2}+y_{2}^{2}=1, \quad\left(x_{1}+m y_{2}\right) y_{1}=0
$$


La dernière nous dit que ou bien nous avons $y_{1}=0$, donc aussi $x_{2}=0$ et alors les premières nous disent que nous avons comme solutions

$$
x_{1}=\varepsilon, \quad y_{2}=\eta \quad(\varepsilon= \pm 1, \eta= \pm 1)
$$

et toutes les autres variables nulles.

Si $y_{1} \neq 0$ les (11) s'ecrivent

$$
x_{1}=-m y_{2}, \quad m^{2}\left[y_{1}^{2}+y_{2}^{2}\right]=1, \quad y_{1}^{2}+y_{2}^{2}=1
$$

et nous avons une impossibilité si $m^{2} \neq 1$. Nous avons donc le théorème:

La fonction

$$
F^{\prime}=4\left(x_{1}+m y_{2}\right) \quad\left(m^{2} \neq 1,0\right)
$$

possède seulement les quatre points critiques (12), si les $\left(10^{\prime}\right)$ sont vérifiées.

Dans le cas où $k=2$, les $\left(10^{\prime}\right)$ sont toujours vérifiées, done il en résulte que sur les variétés quadratique $\left(2^{\prime}\right)$ la fonction $(13)$ a seulement les quatres points critiques (12).

Supposons que l'on a $m^{2}=1$, par exemple $m=1$. En ce cas il en résulte que la fonction

$$
F=4\left(x_{1}+y_{2}\right)
$$

possède, si les $\left(10^{\prime}\right)$ sont vérifiées, une infinité des points critiques dont toutes les coordonnées sont nulles sauf $x_{1}, x_{2}, y_{1}, y_{2}$ et nous avons

$$
x_{1}=-y_{2}, \quad x_{2}=y_{1}, \quad y_{1}^{2}+y_{2}^{2}=1
$$

et par conséquant la fonctions (14) est une fonction Morse-Bott sur la variété quasiquadratique $V_{2 n-1}$. Dans le cas des variétés quadratiques, les $\left(14^{\prime}\right)$ nous donnent tous les points critiques de la fonction (14).

Supposons que l'on a $k>2$, done la variété n'est pas quadratique, et cherchons s'il y a des solutions pour lesquelles une au moins des variables $x_{0}, y_{0}$ n'est pas nulle. En ce cas en multipliant la prémière (10) par $y_{0}$ et la seconde (10) par $\rightarrow x_{0}$ et en sommant on trouve l'équation

$$
\left[y_{2}-m x_{1}\right] Q+2 m y_{1} P=0
$$

et cette équation s'écrit, en tenant compte des (9)

$$
y_{1}\left[x_{1} y_{2}-m y_{1}^{2}\right)=0
$$


Done la dernière (10) nous dit que l'on $a y_{1}=0$ et les autres équations (9) et (10) s'écrivent

$$
\begin{aligned}
& x_{0}^{2}+y_{0}^{2}+P+2 x_{1}^{2}=2, \quad Q=0, \quad x_{1} y_{2} \neq 0, \\
& x_{0}^{2}+y_{0}^{2}-P+2 y_{2}^{2}=2, \quad P=\varepsilon\left[x_{0}^{2}+y_{0}^{2}\right]^{k / 2}, \quad \varepsilon= \pm 1, \\
& y_{2}\left[2\left(x_{0}^{2}+y_{0}^{2}\right)+k P\right]+m x_{1}\left[2\left(x_{0}^{2}+y_{0}^{2}\right)-k P\right]=0,
\end{aligned}
$$

la dernière s'obtenant en multipliant respectivement par $x_{0}$ et $y_{0}$ et en sommant.

En eliminant $x_{1}, y_{2}$ il en résulte l'équation en $Q^{2}=x_{0}^{2}+y_{0}^{2}$

$$
\left(2-\varrho^{2}+\varepsilon \varrho^{k}\right)\left(2 \varrho^{2}+\varepsilon k \varrho^{k}\right)^{2}-m^{2}\left[2-\varrho^{2}-\varepsilon \varrho^{k}\right]\left(2 \varrho^{2}-\varepsilon k \varrho^{k}\right)^{2}=0 .
$$

Le premier membre de cette équation est positif si $\varepsilon$ est égal à 1 et $m^{2}<1$, donc il n'y a pas des solutions. Si $\varepsilon$ est égal à -1 et $m^{2}<1$ il y a une seule solution

$$
x_{0}^{2}+y_{0}^{2}=\varrho^{2}, \quad[0<\varrho<1)
$$

car $m^{2}$ est une fonction qui decroit de 2 à zéro dans l'intervalle

$$
0<x_{0}^{2}+y_{0}^{2}<1
$$

done $x_{0}, y_{0}$ sont solutions de $\left(15^{\prime \prime}\right)$ et de $Q=0$.

Nous avons le théorème:

La fonction (13) a pour $k>2$ plus que quatres points critiques et ces points sont tous isolés.

Cherehons maintenant à voir quelles sont les indices des points critiques (12) de la fonction (4). Dans le voisinage d'un tel point on peut considerer comme quantités du premier ordre les variables

$$
x_{0}, y_{0}, x_{2}, \ldots, x_{n}, \quad y_{1}, y_{3}, \ldots, y_{n}
$$

et les premières équations (3) nous donnent, en tenant compte seulement des termes jusqu'au second ordre et en supposant $k>2$

$$
\begin{array}{ll}
x_{1}=\varepsilon\left[1-\frac{x_{0}^{2}+y_{0}^{2}}{4}-\frac{x_{2}^{2}+\ldots+x_{n}^{2}}{2}\right] & (\varepsilon= \pm 1) \\
y_{2}=\eta\left[1-\frac{x_{0}^{2}+y_{0}^{2}}{4}-\frac{y_{1}^{2}+y_{3}^{2}+\ldots+y_{n}^{2}}{2}\right] & (\eta= \pm 1)
\end{array}
$$

et la dernière équation (3) nous donne

$$
y_{1}=-\varepsilon \eta x_{2}
$$

abstraction faite des termes du second ordre. 
Done on peut considerer $x_{1}, y_{2}$ comme fonctions de $x_{0}, y_{0}, x_{2}, x_{h}(h \geqslant 3)$ et nous avons pour $F$

$$
F=4 \varepsilon+4 m \eta-(\varepsilon+m \eta)\left(x_{0}^{2}+y_{0}^{2}\right)-2(\varepsilon+m \eta) x_{\mathrm{a}}^{2}-2 \varepsilon x_{j}^{2}-2 m \eta y_{j}^{2} .
$$

Supposons que $0<m<1$. En ce cas les points $\varepsilon=-1, \eta=-1$ et $\varepsilon=1, \eta=1$ sont le minimum et le maximum et nous avons, respectivement

$$
\begin{aligned}
& F=-4(1+m)+[1+m]\left(x_{0}^{2}+y_{0}^{2}+2 x_{2}^{2}\right)+2 x_{j}^{2}+2 m y_{j}^{2} \\
& F=4(1+m)-[1+m]\left(x_{0}^{2}+y_{0}^{2}+2 x_{2}^{2}\right)-2 x_{j}^{2}-2 m y_{j}^{2} .
\end{aligned}
$$

Dans le point $R(\varepsilon=-1, \eta=1)$ nous avons

$$
F=-4(1-m)+(1-m)\left[x_{\mathbf{0}}^{2}+y_{0}^{2}+2 x_{2}^{2}\right]+2 x_{h}^{2}-2 m y_{h}^{2}
$$

et le point est l'indice $n-2$, tandis que dans le point $S(\varepsilon=1, \eta=-1)$, nous avons

$$
F=4(1-m)-(1-m)\left[x_{0}^{2}+y_{0}^{2}+x_{2}^{2}\right]-2 x_{h}^{2}+2 m y_{h}^{2}
$$

et il est d'indice $n+1$.

Donc nous avons le théorème:

\section{La fonction}

$$
F^{\prime}=4 x_{1}+4 m y_{1} \quad(0<m<1)
$$

est une fonction ayant sur l'espace $V_{2 n-1}$ avec $k>2$ quatre points critiques non dégénérés, le minimum, le maximum et les deux points $R, S$ d'indices $n-2$ et $n+1$, la fonction ayant comme minimum $-4(1+m)$, comme maximum $4(1+m)$ et ayant dans les points $R$ et $S$ respectivement les valeurs $4(-1+m)$ et $4(1-m)$.

Si $k=2$ la fonction (21) a seulement les points critiques (12) et les formules (16) s'écrivent, en tenant compte des $\left(16^{\prime}\right)$

$$
x_{1}=\varepsilon\left[1-\frac{x_{0}^{2}+x_{2}^{2}+x_{h}^{2}}{2}\right], \quad y_{2}=\eta\left[1-\frac{y_{0}^{2}+x_{2}^{2}+y_{h}^{2}}{2}\right], \quad(h>2)
$$

et nous avons

$$
F=4 \varepsilon+4 \eta m-2 \varepsilon\left[x_{0}^{2}+x_{h}^{2}\right]-2(\varepsilon+m \eta) x_{2}^{2}-2 m \eta\left(y_{0}^{2}+y_{h}^{2}\right)
$$

ce qui nous dit que les points $R$ et $S$ sont d'indices $n-1$ et $n$. 
Le fait que la fonction (4) pour $a=0$ et $m^{2} \neq 1$ possède pour $k=2$ seulement quatre points critiques non dégénérés peut s'exprimer en disant que cette fonction est une bonne fonction de Morse pour les espaces quadratiques $\left(2^{\prime}\right)$. En effet, il est peu probable que l'on puisse avoir en ce cas des fonotions ayant seulement trois points critiques et on ne peut pas avoir des fonctions ayant deux points critiques, car d'après le théorème de $\operatorname{REEB}[3]$, il en résulterait que $\left(2^{\prime}\right)$ est une sphère, ce qu'on sait qu'il n'est pas le cas même pour $k$ pair plus grand que 2 . Le fait que pour $k$ impaire la fonction (4) a plus que quatre points critiques, et l'on sait qu'en cas ce il doit en exister des fonctions ayant seulement deux points critiques, on pent dire que (4) pour $a=0$ n'est pas une bonne fonction de Morse au moins pour $k$ impaire.

Nous allons considérer des fonctions (4) avec $a \neq 0$ et $m=1$. Done en ce cas les conditions $\left(10^{\prime}\right)$ ne peuvent pas plus être vérifiées et par conséquent nous avons toujours

$$
x_{0}^{2}+y_{0}^{2} \neq 0 \text {. }
$$

En multipliant la première (10) avec $y_{0}$ et la seconde avec $-x_{0}$ et en sommant, on obtient en tenant compte de $\left(2^{\prime \prime}\right)$

$$
k\left(y_{2}-x_{1}\right) Q+2 k y_{1} P=2 a y_{0}\left[x_{1} y_{2}-y_{1}^{2}\right] .
$$

D'autre part les (9) nous donnent

$$
P=y_{2}^{2}-x_{1}^{2}, \quad Q=-2 y_{1}\left(x_{1}+y_{2}\right)
$$

et l'équation (22) devient

$$
y_{0}=0 .
$$

D'autre part la dernière (9) nous donne $y_{1}=0$, car on ne peut pas avoir $y_{2}=-x_{1}$, donc $P=0$, car $x_{0}$ est différent de zéro.

Nous avons ainsi le théorème:

Les points critiques de la fonction

$$
f=4\left(x_{1}+y_{2}\right)+2 a x_{0}
$$

ont les coordonnées $y_{0}, x_{2}, y_{1}, x_{h}, y_{h}(h>2)$ nulles, tandisque chaoune des coordonnées $x_{0}, x_{1}, y_{2}$ est différente de zéro.

On peut done écrire les équations (9) et (10) sous la forme

$$
\begin{aligned}
& x_{0}^{2}+x_{0}^{k}+2 x_{1}^{2}=2, \quad x_{0} \neq 0 \\
& x_{0}^{2}-x_{0}^{k}+2 y_{2}^{2}=2, \quad x_{1} y_{2} \neq 0 \\
& x_{0} y_{2}\left[2+k x_{0}^{k-2}\right]+x_{0} x_{1}\left[2-k x_{0}^{k-2}\right]=2 a x_{1} y_{2}
\end{aligned}
$$

et il s'agit de trouver les solutions de ces équations. 
En éliminant $x_{1}, y_{2}$ on trouve pour $x_{0}$ l'équation

$$
\begin{aligned}
{\left[x_{0}^{2}\left[2+k x_{0}^{k-2}\right]^{2}\right.} & {\left[2-x_{0}^{2}+x_{0}^{k}\right]+x_{0}^{2}\left[2-k x_{0}^{k-2}\right]^{2}\left[2-x_{0}^{2}-x_{0}^{k}\right]-} \\
& \left.-2 a^{2}\left[\left(2-x_{0}^{2}\right)^{2}-x_{0}^{2 k}\right]\right]^{2}-4 x_{0}^{k}\left[\left(2-x_{0}^{2}\right)^{2}-x_{0}^{2 k}\right]\left[k^{2} x_{0}^{2 k-4}-4\right]^{2}=0 .
\end{aligned}
$$

Nous avons done une équation en $x_{0}^{2}$ du degré $3 k-2$. Comme les premières (24) nous disent que $x_{0}^{2}$ doit se trouver dans l'intervalle

$$
0<x_{0}^{2}<1
$$

pour obtenir des valeurs réelles pour $x_{1}, x_{2}$ il faut chercher les solutions de (25) dans l'intervalle $\left(25^{\prime}\right)$. Comme dans les extrémités de cet intervalle le premier membre de (25) est positif, il en résulte que l'on a au moins deux solutions de l'équation (25)

$$
x_{0}^{2}=\alpha^{2}, \quad x_{0}^{2}=\alpha^{\prime 2}, \quad 0<\alpha<\alpha^{\prime}<1
$$

et par conséquent au moins quatre solutions pour $x_{0}$, qui conduisent au moins à 8 solutions pour le système (24). Le nombre se réduit à moitié si $x_{0}^{2}=\alpha^{2}$ est une racine double de (25), ce qui arrive si $\alpha^{2}$ est solution de l'équation

$$
k^{2} x_{0}^{2 k-4}-4=0
$$

done si $\alpha$ est solution de l'équation

$$
k \alpha^{k-2}-2=0
$$

équation qui est une identité pour $k=2$. Nous allos supposer donc $k>2$. En supposant a positif, la dernière équation nous dit que l'on doit avoir

$$
a=\frac{2 \alpha}{\beta}, \quad \beta=\sqrt{1-\alpha^{2}\left(\frac{1}{2}+\frac{1}{k}\right)} .
$$

Nous avons done pour $x_{0}$ positif et $k$ impair les deux points critiques

$$
x_{0}=\alpha, \quad x_{1}=\beta, \quad y_{2}=\eta \gamma, \quad \gamma=\sqrt{1-\alpha^{2}\left(\frac{1}{2}-\frac{1}{k}\right)}, \quad \eta= \pm 1
$$

et pour $x_{0}$ négatif les deux points critiques

$$
x_{0}=-\alpha, \quad x_{1}=\varepsilon \gamma, \quad y_{2}=-\beta, \quad \varepsilon= \pm 1
$$

Pour $k$ pair nous avons les quatres points critiques

$$
x_{0}=\alpha, \quad x_{1}=\beta, \quad y_{2}=\eta \gamma ; \quad x_{0}=-\alpha, \quad x_{1}=-\beta, \quad y_{2}=\varepsilon \gamma
$$


Nous voulons maintenant montrer qu'il n'y a pas d'autres points eritiques. Pour cela observons qu'en supposant que $x_{0}= \pm \alpha$ étant une racine de (25) où $\alpha$ est la solution réelle positive de (26), il faut que le premier membre de (25) soit divisible par $\left(x_{0}^{2}-\alpha^{2}\right)^{2}$, donc il faut que nous ayaons

$$
x_{0}^{2}\left[2-x_{0}^{2}\right]\left[4+k^{2} x_{0}^{2 k-4}\right]+4 k x_{0}^{2 k}-a^{2}\left[\left(2-x_{0}^{2}\right)^{2}-x_{0}^{2 k}\right]=\left(x_{0}^{2}-\alpha^{2}\right) R\left(x_{0}^{2}\right)
$$

où $R\left(x_{0}^{2}\right)$ est un polynome en $x_{0}^{2}$ de degré $k-1$. Or ce polynome ne peut pas s'annuler dans l'intervalle (25) car en écrivant le premier membre de $\left(29^{\prime}\right)$ sous la forme

$$
\left(2-x_{0}^{2}\right)\left[\frac{x_{0}^{2 k}\left[a^{2}+4 k\right]}{2-x_{0}^{2}}+k^{2} x_{0}^{2 k-2}+\left(a^{2}+4\right) x_{0}^{2}-2 a^{2}\right]
$$

la fonction entre grandes parenthèses est une fonction croissante dans l'intervalle $\left(25^{\prime}\right)$, donc elle ne peut avoir que la racine $\alpha^{2}$, done $R\left(x_{0}^{2}\right)$ ne s'annule pas et il est toujours positif.

De même nous pouvons écrire

$$
k^{2} x_{0}^{2 k-4}-4=\left(x_{0}^{2}-\alpha^{2}\right) S
$$

où $S$ est aussi un polynome en $x_{0}^{2}$ toujours positif. Donc en divisant l'équation (25) $\operatorname{par}\left(x_{0}^{2}-\alpha^{2}\right)^{2}$ on obtient

$$
R^{2}-x_{0}^{4}\left[\left(2-x_{0}^{2}\right)^{2}-x_{0}^{2 k}\right] S^{2}=0
$$

équation qu'on peut encore écrire

$$
x_{0}^{2 k+4} S^{2}+\left[R+x_{0}^{2}\left(2-x_{0}^{2}\right) S\right]\left[R-x_{0}^{2}\left[2-x_{0}^{2}\right] S\right]=0 .
$$

On voit que le premier terme de cette équation est positif et il en est de même de la première parenthèse dans l'intervalle $\left(25^{\prime}\right)$.

Il reste à montrer qu'il en est de même pour la quantité

$$
\mathcal{M}=R-x_{0}^{2}\left[2-x_{0}^{2}\right] S .
$$

Pour cela multiplions avec $x_{0}^{2}-\alpha^{2}$. Nous avons en vertu des $\left(29^{\prime}\right)$ et (30)

$$
\mathcal{M}\left(x_{0}^{2}-\alpha^{2}\right)=\left[\frac{\left(a^{2}+4 k\right) x_{0}^{2 k}}{2-x_{0}^{2}}+\left(8+a^{2}\right) x_{0}^{2}-2 a^{2}\right]
$$

et la quantité entre grandes parenthèses crôt dans l'intervalle $\left(25^{\prime}\right)$, done $\mathcal{H}$ est positif dans cet intervalle, sauf pour $x_{0}^{2}=\alpha^{2}$, quand il peut être nul. 
Il en résulte que le premier membre de $\left(30^{\prime}\right)$ est toujours positif et nous avons le théorème:

La fonction

$$
f=4\left(x_{1}+y_{2}\right)+\frac{2 \alpha}{\beta} x_{0}, \quad k \alpha^{k-2}=2, \quad \beta=\sqrt{1-\alpha^{2}\left(\frac{1}{2}+\frac{1}{k}\right)}
$$

est pour les variétés exotiques $V_{2 n-1}$ une fonction de Morse ayant seulement les quatre points critiques (28) et $\left(28^{\prime}\right)$ ò̀ $\left(28^{\prime \prime}\right)$ suivant que le nombre $k$ est impair où pair.

Cherchons maintenant à voir quelles sont les indices de ces points critiques. Pour cela supposons que pour les deux points (28) on pose

$$
x_{0}=\alpha+x, \quad x_{1}=\beta+u, \quad y_{2}=\eta \gamma+v
$$

en considérant $x, u, v, y_{0}, x_{2}, y_{1}, x_{h}, y_{h}(h>2)$ comme des quantités du premier ordre. En ce cas les (3) s'écrivent, en tenant compte seulement des termes jusqu'au second ordre et en tenant compte que les termes d'ordre zéro sont nuls et que $\alpha$ satisfait à l'équation (26)

$$
\begin{aligned}
& 4 \alpha x+4 \beta u-(k-2) y_{0}^{2}+k v x^{2}+2\left[u^{2}+x_{2}^{2}+x_{h}^{2}\right]=0 \\
& 4 \eta \gamma v+k y_{0}^{2}-(k-2) x^{2}+2\left(v^{2}+y_{1}^{2}+y_{h}^{2}\right)=0 \\
& \alpha y_{0}+\beta y_{1}+\eta \gamma x_{2}+u y_{1}+v x_{2}+x_{h} y_{h}=0 \quad(h=3, \ldots, n) .
\end{aligned}
$$

En utilisant la dernière de ces équations, les premières nous donnent $u, v$ par les formules, en utilisant aussi (27)

$$
\begin{aligned}
& 4 u=-2 a x-\left(k+\frac{a^{2}}{2}\right) \frac{x^{2}}{\beta}+(k-2) \frac{\left(\beta y_{1}+\eta \gamma x_{2}\right)^{2}}{\beta \alpha^{2}}-\frac{2 x_{2}^{2}}{\beta}-\frac{2 x_{h}^{2}}{\beta}, \\
& 4 v=\frac{k-2}{\gamma} \eta x^{2}-k \eta \frac{\left(\beta y_{1}+\eta x_{2}\right)^{2}}{\gamma \alpha^{2}}-\frac{2 y_{1}^{2}}{\gamma} \eta-\frac{2 y_{h}^{2}}{\nu} \eta .
\end{aligned}
$$

Comme la fonction (23) s'écrit en tenant compte des (28")

$$
f=4(\beta+\eta \gamma)+2 a \alpha+4(u+v)+2 a x
$$

il en résulte que nous avons

$$
f=4(\beta+\eta \gamma)+2 a \alpha-\left[\left(k+\frac{a^{2}}{2}\right)_{\beta}^{1}-\frac{k-2}{\gamma}\right] x^{2}-A-\frac{2}{\beta} x_{h}^{2}-\frac{2 \eta}{\gamma} y_{h}^{2}
$$

où on a posé

$$
A=\frac{2 x_{2}^{2}}{\beta}+\frac{2 \eta y_{1}^{2}}{\gamma}+\left(\frac{k \eta}{\gamma}-\frac{k-2}{\beta}\right) \frac{\left(\beta y_{1}+\eta \gamma x_{2}\right)^{2}}{\alpha^{2}} .
$$


Le discriminant de cette forme quadratique s'écrit

$$
\mathfrak{D}=\frac{2}{\alpha^{2} \beta \gamma}\left[2 \eta \alpha^{2}+\beta^{2} k \eta-(k-2) \gamma^{2}+2 \beta \gamma\right]
$$

et pour $\eta=1$ nous avons

$$
D=\frac{4(\beta+\gamma)}{\alpha^{2} \beta}
$$

done ce discriminant est positif.

Comme nous avons $\gamma>\beta$ il en résulte que pour $\eta=1$ le coefficient de $x^{2}$ est négatif, done le point critique (28) avec $\eta=1$ est un maximum de $F$.

Si $\eta=-1$ le coefficient de $x^{2}$ est négatif mais $A$ est d'indice 1 donc le point critique (28) pour $\eta=-1$ est non dégénére et est d'indice $n$.

Considérons maintenant les points critiques $\left(28^{\prime}\right)$. En posant

$$
x_{0}=-\alpha+x^{\prime}, \quad x_{1}=\varepsilon \gamma+u^{\prime}, \quad y_{2}=-\beta+v^{\prime}
$$

on peut considérer autour d'un tel point comme quantités du premier ordre $x^{\prime}, u^{\prime}, v^{\prime}$, $y_{0}, x_{2}, y_{1}, x_{h}, y_{h}(h>2)$ et les équations (3) s'écrivent, en tenant compte seulement des termes jusqu'au second ordre

$$
\begin{aligned}
& 4 \varepsilon \gamma u^{\prime}-(k-2) x^{\prime 2}+k y_{0}^{2}+2 x_{2}^{2}+2 x_{h}^{2}=0, \\
& -4 \alpha x^{\prime}-4 \beta v^{\prime}+k x^{\prime 2}-(k-2) y_{0}^{2}+2 v^{\prime 2}+2 y_{1}^{2}+2 y_{h}^{2}=0, \\
& \alpha y_{0}+\varepsilon \gamma y_{1}+\beta x_{2}+u^{\prime} u_{1}+v^{\prime} x_{2}+x_{h} y_{h}=0,
\end{aligned}
$$

ce qui nous donne

$$
\begin{aligned}
& 4 u^{\prime}=\varepsilon \frac{k-2}{\gamma} x^{\prime 2}-\eta \frac{k}{\gamma} y_{0}^{2}-\eta \frac{2 x_{2}^{2}}{\gamma}-\eta \frac{2 x_{h}^{2}}{\gamma}, \\
& 4 v^{\prime}=-2 a x^{\prime}+\frac{k x^{\prime 2}}{\beta}-\frac{k-2}{\beta} y_{0}^{2}+\frac{a^{2}}{2} x^{\prime 2}+\frac{2 y_{1}^{2}}{\beta}+\frac{2 y_{h}^{2}}{\beta} .
\end{aligned}
$$

Il en résulte que la fonction $f$ s'écrit

$$
f=4(\varepsilon \gamma-\beta)-2 a \alpha+x^{\prime 2}\left[\frac{k}{\beta}+\frac{a^{2}}{2}+\varepsilon \frac{k-2}{\gamma}\right]+A^{\prime}-\frac{2 \varepsilon}{\gamma} x_{h}^{2}+\frac{2 y_{h}^{2}}{\beta}
$$

où l'on a posé

$$
A^{\prime}=\left(\frac{2-k}{\beta}-\frac{k \varepsilon}{\gamma}\right) \frac{\left(\beta x_{2}+\varepsilon \gamma y_{1}\right)^{2}}{\alpha^{2}}+\frac{2 y_{1}^{2}}{\beta}-\frac{2 \varepsilon}{\gamma} x_{2}^{2}
$$


Pour $\varepsilon=-1$ nous avons un minimum car $A^{\prime}$ est définie positive. Pour $\varepsilon=1$, $A^{\prime}$ est d'indice 1 et est le point critique est d'indice $n-1$.

Supposons maintenant $k$ pair, done que les points critiques sont donnés par les formules (29). Les premiers deux points critiques coincident avec les points critiques (28), done nous atons un maximum pour $n=1$ et un point d'indice $n$ pour $\eta=-1$. Pour avoir les indices des deux autres points critiques (29) posons

$$
x_{0}=-\alpha+\xi, \quad x_{1}=-\beta+\sigma, \quad y_{2}=\varepsilon \gamma+\lambda .
$$

En introduisant dans les formules (3) et en considérant $\xi, \sigma, \lambda, y_{0}, x_{2}, y_{1}, x_{h}, y_{h}$ comme des quantités du premier ordre, nous obtenons en tenant compte seulement des termes jusqu'au second ordre

$$
\begin{aligned}
& -4 \alpha \xi-4 \beta \sigma+k \xi^{2}+(2-k) y_{0}^{2}+2 \sigma^{2}+2 x_{2}^{2}+2 x_{h}^{2}=0 \\
& 4 \varepsilon \gamma \lambda+(2-k) \xi^{2}+k y_{0}^{2}+2 y_{1}^{2}+2 y_{h}^{2}=0 \\
& -\alpha y_{0}-\beta y_{1}+\varepsilon \gamma x_{2}+x_{h} y_{h}=0, \quad(h=3, \ldots, n)
\end{aligned}
$$

de façon que nous arons

$$
\begin{aligned}
& 4 \sigma=-\frac{4 \alpha}{\beta} \xi+\frac{k \xi^{2}}{\beta}+\frac{(2-k) y_{0}^{2}}{\beta}+\frac{2 \alpha^{2}}{\beta^{3}} \xi^{2}+\frac{2 x_{2}^{2}}{\beta}+\frac{2 x_{h}^{2}}{\beta} \\
& 4 \lambda=-\frac{(2-k) \varepsilon}{\gamma}-\frac{\varepsilon k}{\gamma} y_{0}^{2}-\frac{2 \varepsilon y_{1}^{2}}{\gamma}-\frac{2 \varepsilon y_{h}^{2}}{\gamma}
\end{aligned}
$$

où on doit poser au lieu de $y_{0}$ la quantité $\left(\beta y_{1}-\varepsilon \gamma x_{2}\right) / \alpha$. Il en resulte que la fonction $f$ s'écrit

$$
f=4(\varepsilon \gamma-\beta)-4 \frac{\alpha^{2}}{\beta}+\xi^{2}\left[\frac{k}{\beta}+\frac{2 \alpha^{2}}{\beta^{3}}+\varepsilon \frac{k-2}{\gamma}\right]+\mathcal{M}+\frac{2 x_{h}^{2}}{\beta}-\frac{2 \varepsilon y_{h}^{2}}{\gamma}
$$

où nous avons posé

$$
\mathcal{H}=\frac{2 x_{2}^{2}}{\beta}-\frac{2 \varepsilon y_{1}^{2}}{\gamma}-\left(\frac{k-2}{\beta}+\frac{\varepsilon k}{\gamma}\right) \frac{\left(\beta y_{1}-\varepsilon \gamma x_{2}\right)^{2}}{\alpha^{2}} .
$$

Il en résulte que le point $\varepsilon=-1$ est le minimum de $f$. Pour $\varepsilon=1$ le coefficient de $\xi^{2}$ est positif. Quant à $\mathcal{H}$ elle est d'indice 1 donc le point (29) avec $\varepsilon=1$ est pour $k$ pair plus grand que deux, aussi un point d'indice $n-1$.

Nous allons observer que dans les points d'indices $n-1$ et $n$ nous avons respectivement comme valeurs de $f$

$$
f_{1}=\frac{4 \gamma \beta-4 \beta^{2}-2 \alpha^{2}}{\beta}, \quad f_{2}=\frac{4 \beta^{2}+2 \alpha^{2}-4 \beta \gamma}{\beta}
$$


et nous avons $f_{1}<0$ et $f_{2}>0$. En effet dire que $f_{1}<0$, revient à dire que nous avons

$$
4 \gamma \beta<4 \beta^{2}+2 \alpha^{2}=4-\frac{4 \alpha^{2}}{k}
$$

En elevant au carré, il en résulte que l'on doit avoir

$$
\alpha^{2}<\frac{4 k(k-2)}{k^{2}-8} \text {. }
$$

En tenant compte de (26) il en résulte que l'on doit avoir

$$
4^{k-3} \frac{k^{2} k^{k-2}(k-2)^{k-2}}{\left(k^{2}-8\right)^{k-2}}>1
$$

ce qui a evidemment lieu car dans le premier membre nous avons le rapport d'un polynome de dégré $2 k-2$ dans $k$ et d'un polynome du dégré $2 k-4$.

Supposons maintenant $k=2$. En ce cas l'équation (26) est une identité et les points critiques de $f$ satisfont aux équations

$$
2 x_{0}=a x_{1}, \quad x_{0}^{2}+x_{1}^{2}=1, \quad y_{2}^{2}=1, \quad x_{1} y_{2} \neq 0 .
$$

En supposant $a=2 \operatorname{tg} \varphi$, donc en prenant comme fonction

$$
f=4\left[x_{1}+y_{2}+\operatorname{tg} \varphi x_{0}\right)
$$

où $\varphi$ est une constante, les coordonnées des points critiques sont toutes nulles sauf

$$
x_{0}=\varepsilon \sin \varphi, \quad x_{1}=\varepsilon \cos \varphi, \quad y_{2}=\eta \quad(\varepsilon= \pm 1, \eta= \pm 1) .
$$

En supposant que nous avons $0<\varphi<\pi / 2$ donc $\cos \varphi>0, a>0$ et en posant

$$
x_{0}=\varepsilon \sin \varphi+x, \quad x_{1}=\varepsilon \cos \varphi+u, \quad y_{2}=\eta+y
$$

les équations $\left(2^{\prime}\right)$ nous donnent

$$
\begin{aligned}
& 2 n=-2 x \operatorname{tg} \varphi-\frac{\varepsilon x^{2}}{\cos \varphi}-\frac{\varepsilon x_{2}^{2}}{\cos \varphi}-\frac{\varepsilon x_{h}^{2}}{\cos \varphi}, \\
& 2 y=-\eta \frac{\left(\varepsilon \sin \varphi y_{0}+\eta x_{2}\right)^{2}}{\cos ^{2} \varphi}-\eta y_{h}^{2} \quad(h=3, \ldots, n) .
\end{aligned}
$$

En introduisant dans (40) on trouve que pour $\varepsilon=\eta=-1$ nous avons un minimum, pour $\varepsilon=-1, \eta=1$ un point d'indice $n-1$, pour $\varepsilon=1, \eta=-1$ un point 
d'indice $n$ et pour $\varepsilon=\eta=1$ un maximum. Il en résulte que pour $k=2$ il y a une infinités de fonctions (40) ayant seulement quatres points critiques d'indices 0 , $n-1, n, 2 n-1$.

Nous avons donc le théorème:

Si $k$ est impair les quatre points critiques $(28),\left(28^{\prime}\right)$ de la fonction (23) sont le maximum et le minimum pour $\eta=1$ et $\varepsilon=-1$ et des points critiques non dégénérés d'indices respectivement $n-1$ et $n$ pour $\varepsilon=1$ et $\eta=-1$, la fonction ayant dans ces points tes valeurs

$$
4(\gamma-\beta)-\frac{4 \alpha^{2}}{\beta}, \quad 4(\beta-\gamma)+\frac{4 \alpha^{2}}{\beta}
$$

Le même théorème a lieu si $k$ est pair et plus grand que 2 pour les points critiques (29). Si $k=2$, e'est sont le fonctions (40) qui ont quatres points critiques ayant respectivement les indices $0, n-1, n, 2 n-1$. On peut observer aussi que pour $k$ impair une transformation de la forme

$$
x_{0}^{\prime}=-x_{0}, \quad y_{0}^{\prime}=-y_{0}, \quad x_{1}^{\prime}=-y_{2}, \quad x_{2}^{\prime}=y_{s}, \quad x_{h}^{\prime}=y_{k}, \quad y_{k}^{\prime}=-x_{h}
$$

laisse la variété $V_{2 n-1}$ invariante et change les points critiques d'indice $n-1$ et $n$ entre eux et de même le maximum en minimum, car la fonction change de signe.

Dans le cas pair c'est la transformation

$$
x_{i}^{\prime}=-x_{i}, \quad y_{i}^{\prime}=-y_{i}
$$

qui change entre eux les points critiques.

On peut terminer, en observant, qu'en tenant compte du théorème de Reeb, qui dit que sur une sphère, on peut construire une fonction de Morse ayant seulement deux points critiques, il fallait trouver une telle fonction dans le cas où $k$ est impair, mais je n'ai pas reussi a faire cela.

\section{BIBLIOGRAPHIE}

[1] F. Hirzebruch, Singularites and exotic spheres, Sòminaire Bourbaki (novembre 1966), pp. 314-331.

[2] G. VRAnCeAnd, Qualche proprietà differenziale delle sfere exotiche, Rendiconti di Milano, (1968).

[3] G. REEB, Sur certaines propriétés topologiques des variétés feuilletées, Hermann, Parir, 1952 , p. 147. 